Europhysics Letters

PREPRINT

\title{
Dynamic regimes of hydrodynamically coupled self-propelling particles
}

\author{
IsAaC Llopis $^{1}$ and Ignacio PAgOnABARRAGA ${ }^{1}$ \\ 1 Departament de Física Fonamental, Universitat de Barcelona, C. Martí i Franqués 1, \\ 08028 Barcelona, Spain
}

PACS. 87.18.Hf - Pattern formation.

PACS. 05.10.-a - Computational methods in statistical physics and nonlinear dynamics.

PACS. 82.70.Dd - Colloids.

PACS. 87.17.-d - Cellular structure and processes.

\begin{abstract}
We analyze the collective dynamics of self-propelling particles (spps) which move at small Reynolds numbers including the hydrodynamic coupling to the suspending solvent through numerical simulations. The velocity distribution functions show marked deviations from Gaussian behavior at short times, and the mean square displacement at long times shows a transition from diffusive to ballistic motion for appropriate driving mechanism at low concentrations. We discuss the structures the spps form at long times and how they correlate to their dynamic behavior.
\end{abstract}

Introduction. - A great variety of microorganisms, such as bacteria, have the ability to displace in a fluid medium. Organisms have developed a number of swimming strategies using for example flagella, cilia, or surface waves [1]. These individuals always produce the force needed to generate their motion. As a result, they are out of thermal equilibrium, which makes their motion to differ qualitatively from the motion of suspensions of passive particles, such as colloids [2]. Active motion is not restricted to living organisms, and is shared by a number of biomolecules; e.g. membrane proteins which move or rotate as a result of the energy they get from ionic pumps. In all these situations, propulsion induces flow in the embedding solvent leading to effective interactions among the active particles. Although it is known how these interactions affect the dynamics of passive suspensions, for example colloids, the role they play in self-propelling particles (spps) suspensions remains to be understood.

Despite substantial effort and progress have improved the understanding and characterization of self propulsion and how it emerges from the elementary molecular mechanisms [1], the implications and characterization of this motion on collective scales remain far less well understood. Although the hydrodynamics of microorganisms has been addressed [3], with special emphasis on bioconvection [4], the relation of this hydrodynamic behavior with the detailed properties of spps remains a challenge. Recent experimental evidence shows the need of a detailed understanding to explain the collective particle motion at small scales induced by hydrodynamics [5], or to describe beads' diffusion in bacteria suspensions [6]. Such insight is

(c) EDP Sciences 
also useful to clarify the relevance of spps' orientation on their rheological response [7]. Theoretical studies have analyzed the role spps' interactions have in the emergence of collective behavior (such as flocking) and have provided a framework to understand their properties [8], although disregarding hydrodynamic interactions.

In this letter we address the different dynamical regimes which characterize spps suspensions. We will focus on the relevant hydrodynamic couplings at the relevant time scales; an analysis analogous to the one carried out to characterize the dynamics of passive suspensions [9]. We will disregard any coupling other than hydrodynamic and excluded volume to focus on their specific relevance to collective propulsion.

Model. - We will consider a lattice Boltzmann (LB) model to describe the fluid and address the appropriate time scales which determine the coupling of the active particles and the fluid. The state of the fluid is specified by the average number of fluid molecules, $n(\mathbf{c}, \mathbf{r}, t)$, with velocity $\mathbf{c}$, at each lattice site, $\mathbf{r}$. The time evolution of $n(\mathbf{c}, \mathbf{r}, t)$ is described by the discretized analog of the linearized Boltzmann equation [10], involving propagation and collision. Collisions are specified in order that the hydrodynamic fields satisfy the Navier-Stokes equation at large scales. We will use units so that the mass of the lattice molecule, the lattice spacing and the time step are unity, the kinematic viscosity $\nu$ of the fluid will be smaller than 1 and the speed of sound $c_{s}=1 / \sqrt{2}$; the density $\rho$ is constant for practical purposes. The suspended, spherical, particles of radius $R=5 / 2$ are modeled by the set of boundary links which join fluid nodes and nodes interior to the particles [11]. Stick boundary conditions are applied through appropriate kinetic rules on the boundary links which determine the force the fluid exerts on the particle; the equations of motion of the particles are then integrated using the self-consistent method described in Ref. [12]. Whenever two particles are at a distance slightly larger than their diameter, an elastic collision is performed to ensure they do not overlap.

Since we are interested in the basic mechanisms controlling the collective dynamics of spps' suspensions, we introduce a simplified self-propulsion mechanism, complementary to others proposed in the literature [13]. We subtract a constant amount of momentum with fixed magnitude $\Delta p_{0}$, uniformly distributed over all the fluid nodes connected to solid nodes and which lie within a cone of angle $\psi_{0}$ around a predefined direction of motion. The value of $\psi_{0}$ affects the details of the local flow field, but does not modify the collective features we will analyze; for simplicity's sake we have chosen $\psi_{0}=\pi / 2$. The direction of motion is determined at the startup and moves rigidly with the propeller [14]; in particular a spp rotation leads to the corresponding modification in its propulsion direction. The subtracted momentum is added to the spp, preserving momentum conservation. Since we are interested in the active motion of small organisms and colloids, $\Delta p_{0}$ is chosen to ensure that inertia is negligible [15]. A particle released from rest will reach a constant velocity inducing a dipolar flow in the embedding fluid at long distances. Even if this mechanism is inspired by internal peristaltic motion [16], we will concentrate on generic aspects of propulsion. To this end, we will address the relevance of the symmetry of the fluid flow surrounding the spps by comparing this asymmetric driving, with a second symmetric propelling mechanism. In order to focus on the basic mechanisms generated by the effective dissipative interactions induced by the fluid we disregard thermal fluctuations.

Short time dynamics. - A spp of radius $R$ released from rest in a fluid of viscosity $\nu$ will start moving and reach a terminal constant velocity, $u_{\infty}$, on a characteristic time $\tau_{r} \sim R^{2} / \nu$ in which the fluid flow induced by the spp is established. Consistent with this hydrodynamic origin, we have verified that the asymptotic velocity is reached algebraically, controlled by the long-time tail, $u(\tau)-u_{\infty} \sim \tau^{-d / 2}(d>1), d$ being the system's dimensionality. The asymptotic 

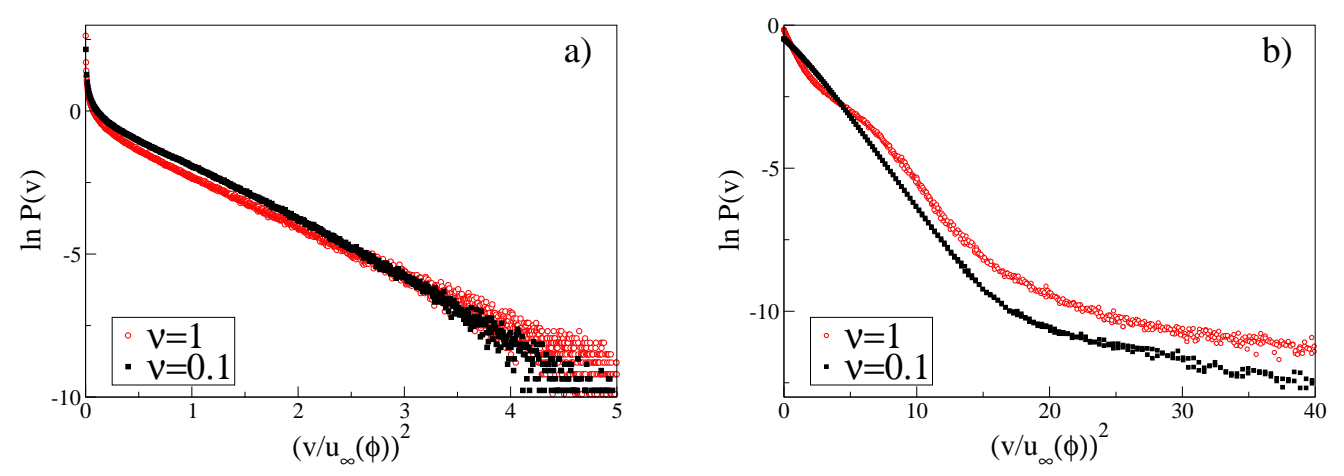

Fig. 1 - Velocity distribution functions at volume fraction $\phi=0.02$ in a system of $N=1020$ spps. Velocities are collected from independent initial conditions at $\tau / \tau_{r} \sim 2$. a) Particle velocity distribution function; b) fluid velocity distribution function.

velocity arises from a balance between the driving force and the friction, $u_{\infty}=\Delta p_{0} /(12 \pi \eta R)$, where $\eta=\rho \nu$ is the solvent viscosity [14].

The induced flows generated by the propellers have a strong influence on their velocity distributions; the non-equilibrium character of their motion leads to velocity distributions that depart markedly from a Gaussian, as displayed in Fig. 1.a. The velocity distribution function, $P(v)$, is characterized by an algebraic decay at small velocities, consistent with $P(v) \sim 1 / v$, while its asymptotic decay is consistent with a Gaussian. In Fig. 1 b we show the corresponding fluid velocity distribution; in this case the small velocity regime decays exponentially, followed by a decay compatible with a Gaussian in the region of velocities of the order of $u_{\infty}$ before it develops an asymptotic decay characterized by a generalized exponential $P(v) \sim \exp \left[-\left(v / v_{0}\right)^{\alpha}\right]$ with exponent $\alpha=0.4 \pm 0.2$ [17]. Although deviations from Gaussian behavior are known in other non-equilibrium systems, e.g. driven granular fluids [18], in spps suspensions they arise due to coupling to the collective motion of the embedding solvent.

Interactions among spps modify their effective mean asymptotic velocity at time scales $\tau_{r}$, as depicted in Fig. 2. where averages over 20 independent configurations have been carried out. Spps with parallel orientations interact more strongly than their randomly moving counterparts. The former situation is reminiscent of sedimentation in passive colloids and a quantitative comparison, as displayed in Fig. 2 shows that the absence of a net applied external force leads to a weaker coupling, due to the dipolar nature of the induced velocity flow [19].

Long time dynamics. - For the small Re of interest, spps' propulsion leads to configurational changes on time scales $\tau_{m} \sim R / u_{\infty}>\tau_{r}$, when spps will also modify their motion through collisions. We have observed that angular velocity autocorrelation functions decay on $\tau_{m}$; hence spps modify their direction of motion mostly through collisions. Although additional mechanisms which affect the spps' direction of motion, such as tumbling, play a relevant role in the decorrelation of particles' orientation, most of the features described in this manuscript survive qualitatively if they are accounted for [14]. At $\tau>\tau_{m}$ we enter in the long-time dynamics regime of spps suspensions. In order to reach this long-time regime, we have performed computer simulations of the LB model in two dimensions [20]. Fig. 3 displays in circles the mean square displacement (msd) at an intermediate volume fraction, 

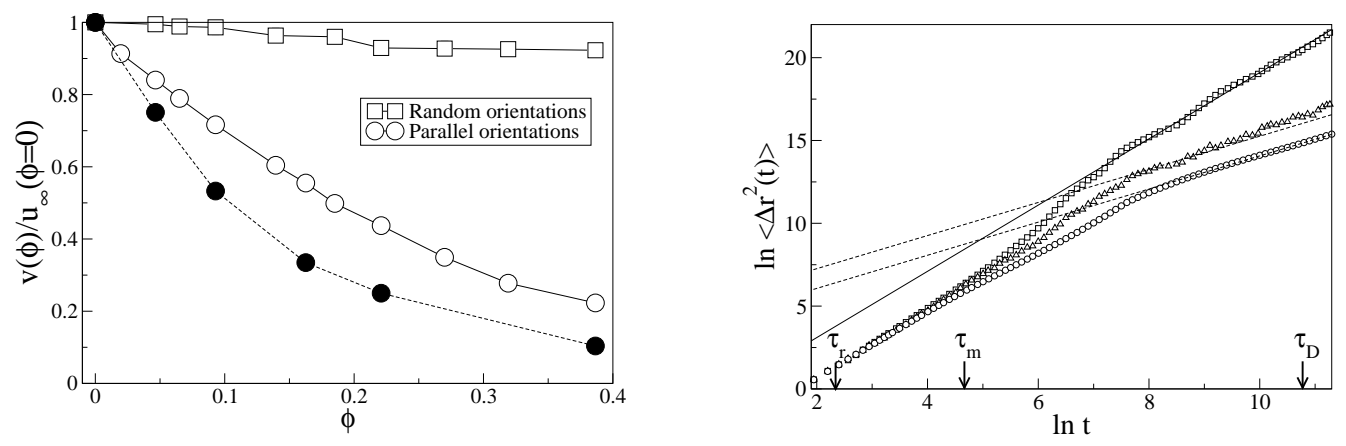

Fig. 2 - Dependence of spps' velocities on volume fraction for random and parallel orientations for a system of $N=400$ spherical particles in a fluid of viscosity $\nu=0.5$. Black circles: sedimentation velocity for hard spheres; it has been obtained with the same model by introducing a uniform external field and setting $\Delta p_{0}=0$.

Fig. 3 - Mean square displacement in a spps' suspension for a two-dimensional system of 400 propellers, kinematic viscosity $\nu=1$ and Reynolds $R e=0.25$. The continuous straight line corresponds to ballistic motion, $\left\langle\Delta r^{2}(t)\right\rangle \sim t^{2}$, while the long-dashed ones correspond to diffusion, $\left\langle\Delta r^{2}(t)\right\rangle \sim t$. Circles correspond to $\phi=0.282$ whereas squares and triangles refer to $\phi=0.1$ for different initial conditions. We display also the characteristic times: $\tau_{r} \sim R^{2} / \nu \sim 10, \tau_{m} \sim R / u_{\infty} \sim 100$ and $\tau_{D} \geq R^{2} / D \sim 5 \cdot 10^{4}$.

$\phi=0.282$, with a crossover from ballistic to diffusive asymptotic behavior at the collision time scale, $\tau \sim \tau_{m}$. At longer times, a diffusive regime is achieved for $\tau_{D} \geq R^{2} / D$, where $D$ is the diffusion coefficient. Since diffusion can only be achieved through succesive collisions and relaxations to the local fluid flows, $\tau_{D}>\tau_{m}$, which implies that the propellers' Péclet number satisfies $P e \equiv u_{\infty} R / D>1$; hence for these systems convection is always a relevant mechanism. This feature is consistent with the long crossover regime observed in the msd of beads in a bacteria suspension [6].

At low volume fractions a different scenario is observed, as displayed in squares and triangles in Fig. 3 In the collision regime, $\tau_{m}$, a clear acceleration of the particles is observed; such behavior leads at longer times to a superdiffusive regime. The observed acceleration at intermediate times is due to the increase in the spps' velocities through hydrodynamic coupling. We have seen that collisions tend to correlate locally spps which move initially at random. This tendency leads to an acceleration and to the formation of large clusters which eventually split into smaller aggregates with a finite life time of the order of $\tau_{m}$. For some initial conditions, we have observed the appearance of system-spanning clusters that lead to long-time ballistic motion. The analysis of the propellers' velocity autocorrelation function (vacf) shows a consistent behavior; after an initial exponential decay at times of order $\tau_{m}$, the vacf increases toward a plateau value at later times. The vacf shows small oscillations in this late regime that we attribute to cluster dynamics. These oscillations are also visible in the mean square displacement in Fig. 3 a.

To gain insight in the structures the propellers develop, we have analyzed their spatial distribution through the generalized radial distribution functions $g_{n}(r) \equiv\left\langle P_{n}\left(\cos \theta_{i j}\right)\right\rangle$, with $\theta_{i j}$ being the relative angle between the direction of motion of the reference particle $i$ and that of all particles $j$ at a distance between $r$ and $r+d r$, where $P_{n}$ is the n-th degree Legendre poly- 

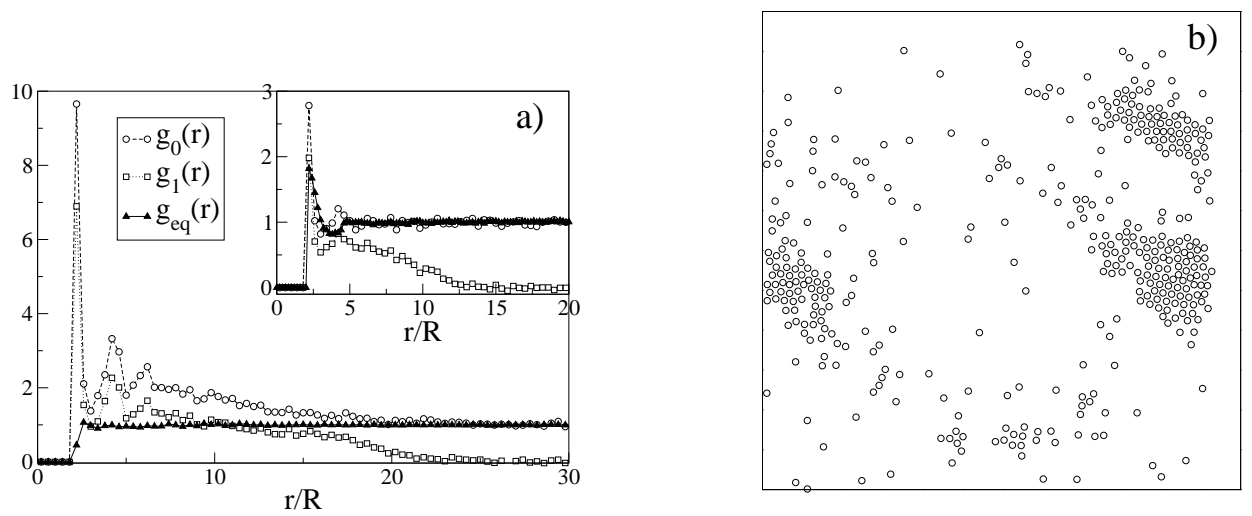

Fig. 4 - a) Radial distribution functions of a spps' suspension for a two-dimensional system of $N=815$, kinematic viscosity $\nu=1$ and Reynolds $R e=0.25$ at times $\tau \geq 10 \tau_{m}$ and area fraction $\phi=0.1$; Inset: Radial distribution functions for an analogous system at $\phi=0.1$, when the driving mechanism is symmetric around the direction of motion. See text for definition of $g_{n}$. Thick black line corresponds to the short time regime, $\tau \sim \tau_{r}$. b) Snapshot of the spps solution with asymmetric driving at $\phi=0.1$ at time $\tau \geq 90 \tau_{m}$.

nomial. Fig. [4 a shows the lowest order radial distribution functions, where simulations over 5 independent initial configurations are used to increase the statistical accuracy. The value of the pair distribution function at contact, $g_{0}(r=2 R)$, is much greater than its equilibrium counterpart, which shows the tendency of the spps to remain closer as a result of their activity. One can see a clear structuring on length scales much larger than the spps' size signalling the formation of mesoscale structures. The decay of $g_{0}(r)$ is consistent with the aggregates observed in simulations; in Fig. 固b we show a typical configuration in the long-time regime. The decay of $g_{1}(r)$ on the same length scale, indicates that there is a local tendency of spps to share common direction, so that the transient aggregates have a common direction of motion. This implies that spherical spps develop orientational order; their dynamics will display similarities with that of non-spherical spps (see e.g. [7]). The observed aggregates form and dissolve, and hence there is no sign of permanent aggregation induced through hydrodynamics. Additional interactions, for example chemical signaling, may stabilize these aggregates. We have also analyzed the velocity distribution functions both for propellers and fluid at long times, $\tau \geq \tau_{D}$, where we have not found significant deviations from Gaussian behavior.

We have also studied the collective behavior of symmetric propellers to assess the relevance of the specificity of the propulsion mechanism. In this case the momentum, $\Delta p_{0}$, is extracted uniformly from all nodes lying within the two cones defined by the angles $\psi_{0}$ and its suplementary, $\pi-\psi_{0}$. For this driving the velocity distributions at short times are equivalent to those found for asymmetrically driven propellers. Asymptotically $\left(\tau \geq \tau_{D}\right)$, we have observed diffusive behavior for all the concentrations explored. Correspondingly, the radial distribution function $g_{0}(r)$ does not show a slow decay on distances larger than $R$, as displayed in the inset of Fig. 4. In this case one do not observe transient, mesoscopic, aggregates. The contact value is still significantly larger than its equilibrium counterpart, although not as large as for asymmetric driving. In this case $g_{1}(r)$ decays to zero on longer length scales than $g_{0}(r)$, which shows a tendency to local orientation of spps, and hence a sensitivity toward nematization, as has been predicted for non-s'herical spps [7]. Therefore, hydrodynamics makes particles approach, while its coupling strength determines the properties of spps' structures. 
EUROPHYSICS LETTERS

Even if we cannot rule out a further relaxation to an asymptotic diffusive regime for asymmetric propulsion at low $\phi$, the observed formation and dissolution of aggregates on the characteristic collision time scale, $\tau_{m}$, and the corresponding oscillations in the vacf strongly suggest that the superdiffusive regime is properly sampled. We have checked that this longtime behavior is robust upon changing the system size and for a variety of volume fractions. We have also seen that the dependence of the msd on volume fraction is consistent; i.e. upon increasing $\phi$ we observe superdiffusive behavior, then a crossover region where the dynamics is intermediate on the simulation time scale which ends up becoming diffusive at higher volume fractions.

The transition to collective motion, characterized by the asymptotic superdiffusive regime, is analogous to reported flocking transitions [8], although the mechanism in this case is dissipative, through hydrodynamics. We have verified that this transition disappears when considering a symmetric propulsion mechanism, and hence attribute flocking as arising from the strength of hydrodynamic coupling due to the drag a moving spp induces on neighboring propellers. Such a dissipative interaction is responsible both for the velocity increase and for spps' alignment observed at $\tau \geq \tau_{m}$. In previous flocking models, the transition to collective macroscopic motion is determined by the effect of interactions on the direction of motion. These interactions are absent in our model, which indicates that hydrodynamic coupling will favor previously observed flocking transitions once the additional interactions between propellers are accounted for, and will promote it at smaller volume fractions.

Conclusions. - We have introduced a new, simple mesoscopic model for self-propulsion which resolves hydrodynamics and have identified the relevant dynamical regimes for low Reynolds numbers spps suspensions. In this model we have been able to carry out a detailed analysis which has allowed us to clarify the role of hydrodynamics on the different time scales.

In particular, we have considered the correlations induced by these dissipative interactions at short times, which lead to clear deviations from Gaussian velocity distributions. Such effects are visible both in the spps and in the fluid velocity distributions. Spps interact through collisions at longer time scales and hydrodynamics can accelerate them and favor the formation of transient aggregates. Depending on the details of the propulsion mechanism, spps may exhibit both diffusive and superdiffusive dynamics at the scales at which structural rearrangements take place. The understanding of these regimes, their connections to flocking transitions and their implications in the collective properties of spps suspensions require a more detailed study.

The authors acknowledge financial support from DGICYT of the Spanish Government, they thank R. Adhikari and M.E. Cates for fruitful discussions, and M.E. Cates for suggestions and a careful reading of the manuscript. I.Ll. wants to thank the HPC-EUROPA project (RII3-CT-2003-506079), with the support of the European Community - Research Infrastructure Action. I.P. thanks Distinció from DURSI (Generalitat de Catalunya) and P.B. Sunil Kumar for fruitful discussions.

\section{REFERENCES}

[1] D. Bray, Cell Movements: From Molecules to Motility (Garland Publishing, New York) 2001.

[2] W. Russel, D.A. Saville and W.R. Schowalter, Colloidal Dispersions (Cambridge University Press, Cambridge) 1989. 
[3] T.J. Pedley and J.O. Kessler, Annu. Rev. Fluid Mech., 24 (313) 1994.

[4] I. Tuval, L. Cisneros, C. Dombrowski, C.W. Wolgemuth, J.O. Kessler and R.E. GoldStein, Proc. Nat. Acad. Sci. USA, 102 (2277) 2005.

[5] I.H. Riedel, K. Kruse and J. Howard, Science, 309 (300) 2005.

[6] X.-L. Wu and A. Libchaber, Phys. Rev. Lett., 84 (3017) 2000.

[7] Y. Hatwalne, S. Ramaswamy, M. Rao and R.A. Simha, Phys. Rev. Lett., 92 (118101) 2004.

[8] J. Toner, Y.H. Tu and S. Ramaswamy, Annals of Physics, 318 (170) 2005.

[9] P.N. Pusey, Liquids, Freezing and the Glass Transition, edited by J.P. HAnsen et al. (Elsevier, Amsterdam) 1991, sect. 10.

[10] S. SuCCI, The Lattice Boltzmann equation for fluid dynamics and beyond (University Press, Oxford) 2001.

[11] A.J.C. Ladd and R. Verberg, J. Stat. Phys., 104 (1191) 2001.

[12] C.P. Lowe, A.J. Masters and D. Frenkel, J. Chem. Phys., 103 (4) 1995.

[13] S. Ramachandran, P.B. Sunil and I. Pagonabarraga, Eur. Phys. J. E , 20 (151) 2006.

[14] I. Llopis and I. Pagonabarraga, preprint.

[15] The Reynolds number $(R e)$ is defined in terms of the particle's velocity and size and using the fluid kinematic shear viscosity. $\Delta p_{0}$ is chosen to ensure that $R e$ never exceeds 0.1 (to assess the role of finite $R e$ in LB, see e.g. M.E. Cates, J.-C. Desplat, P. Stansell, A. Wagner, K. Stratford, R. Adhikari and I. Pagonabarraga, Proc. Royal Soc. London A, 363 (1917) 2005).

[16] A. Ajdari and H. Stone, Phys. Fluids, 11 (1275) 1999.

[17] We cannot achieve velocities beyond $10 u_{\infty}$. Hence, we cannot rule out an additional, asymptotic regime controlling the tails of the velocity distribution. We have checked this exponent both by assuming the functional form and fitting it and by analyzing the logarithmic derivative to extract the exponent (cf. T. Biben, P.A. Martin and J. Piasecki, Physica A, 310 (308) 2002).

[18] Granular gas dynamics, edited by T. Pöschel and N. Brilliantov (Springer, Berlin) 2003.

[19] J.L. Anderson, Ann. Rev. Fluid Mech., 21 (61) 1989.

[20] Preliminary results in three dimensional systems give results consistent with the ones discussed here. 